\title{
Enhanced ion acoustic fluctuations and ion outflows
}

\author{
F. R. E. Forme and D. Fontaine \\ Centre d'étude des Environnements Terrestre et Planétaires, Velizy, France
}

Received: 2 February 1998 / Revised: 2 June 1998 / Accepted: 26 June 1998

\begin{abstract}
A number of observations showing enhanced ion acoustic echoes observed by means of incoherent scatter radars have been reported in the literature. The received power is extremely enhanced by up to 1 or 2 orders of magnitude above usual values, and it is mostly contained in one of the two ion acoustic lines. This spectral asymmetry and the intensity of the received signal cannot be resolved by the standard analysis procedure and often causes its failure. As a result, and in spite of a very clear spectral signature, the analysis is unable to fit the plasma parameters inside the regions of ion acoustic turbulence. We present European Incoherent Scatter radar (EISCAT) observations of large ion outflows associated with the simultaneous occurrence of enhanced ion acoustic echoes. The ion fluxes can reach $10^{14} \mathrm{~m}^{-2} \mathrm{~s}^{-1}$ at $800 \mathrm{~km}$ altitude. From the very clear spectral signatures of these echoes, a method is presented to extract estimates of the electron temperature and the ion drift within the turbulent regions. It is shown that the electron gas is strongly heated up to $11000 \mathrm{~K}$. Also electron temperature gradients of about $0.02 \mathrm{~K} / \mathrm{m}$ exist. Finally, the estimates of the electron temperature and of the ion drift are used to study the possible implications for the plasma transport inside turbulent regions. It is shown that strong electron temperature gradients cause enhancement of the ambipolar electric field and can account for the observed ion outflows.
\end{abstract}

Key words. Ionosphere (auroral ionosphere; ionosphere -magnetosphere interactions; plasma waves and instabilities).

\section{Introduction}

Since the first observations by incoherent scatter radars (ISR) (Foster et al., 1988; Collis et al., 1991; Rietveld et al., 1991) a number of enhanced ion acoustic echoes have been detected subsequently in the auroral ionosphere with a very characteristic signature in the frequency spectrum of the received signal. The received power is extremely enhanced by up to 1 or 2 orders of magnitude above the usual values, and is mostly contained in one of the two ion acoustic lines observed displaced from the transmitter frequency. This spectral asymmetry and the intensity of the received signal cannot be treated by the standard analysis procedure and often causes its failure. As a result, and in spite of a very clear spectral signature, the analysis is unable to fit the plasma parameters inside the regions of ion acoustic turbulence.

The origin of these intense acoustic echoes also remains controversial. Different theories have emerged to explain the triggering of these echoes from various processes: the current-driven instability (Rietveld et al., 1991) the ion-ion drift instability (Wahlund et al., 1992a), or the parametric decay of plasma waves (Forme, 1993). In the absence of direct observations of the ionospheric plasma inside the turbulent regions, these theories first exploit, in various ways, available pieces of information obtained in regions adjacent to the turbulence. In addition, the theories make use of various assumptions about the plasma profiles inside turbulent regions, which remain to be validated, such as the heating rate of the plasma species, the concentration and velocity of minor ion species, the assumed presence of intense field-aligned currents, of parallel electric fields, or of simultaneous plasma waves.

Enhanced ion acoustic fluctuations are associated with enhanced electron temperature (Wahlund et al., 1993), large field-aligned bulk ion outflows from the topside ionosphere (Wahlund et al. 1992b) and the 
presence of red arcs induced by the precipitation of lowenergy particles (several tens or hundreds of $\mathrm{eV}$ ) (Collis et al., 1991). Wahlund et al. (1993) showed that the heating is partly due to precipitating soft electrons in the energy range $100-500 \mathrm{eV}$ and they suggested that the major heat source is due to the turbulence itself. Forme et al. (1993a) presented a model that calculates the effects of low frequency turbulence on the thermal structure of the topside ionosphere. They found a good qualitative agreement assuming that the electrons are heated by the turbulent electric field. For the ion outflows, Wahlund et al. (1992b) suggested that the ions are accelerated by a parallel electric field in the ionosphere. In their interpretation the enhanced fieldaligned electric field is caused by anomalous resistivity due to low-frequency plasma turbulence. They contrasted this type of acceleration mechanism with thermal ion outflows where frictionally heated ions cause strong pressure gradients, which in turn push them upward. However, recent observation showed that ion outflows are not always observed when the ion acoustic instability occurs. Forme et al. (1995) showed that the ion outflows are more likely to be associated with large electron temperatures. However the lack of information about the plasma parameters inside the turbulent regions does not allow us to determine the acceleration mechanism.

Ion outflows are also of a great interest in the scope of ionosphere magnetosphere coupling. The observations of large populations of energetic $\mathrm{O}^{+}$ions in the magnetosphere, obviously of terrestrial origin, reviewed by Johnson et al. (1979), posed the problem of the importance of the ionosphere in refilling the magnetosphere. Chappell et al. (1987) suggested that the ionosphere is the dominant source for ions in the magnetosphere. Low-energy $(<50 \mathrm{eV})$ ion outflows in the high-latitude magnetosphere are $\mathrm{O}^{+}$dominated (Lockwood et al., 1985) and contain a significant molecular ion component which suggests that the outflows originate in the ionospheric $F$ region. Several radar observations of ion outflows (Farmer et al., 1984; Winser et al., 1989; Jones et al., 1988) appear to be the thermal plasma signature of ion injection into the magnetosphere.

We will present (Sect. 2) European Incoherent Scatter radar (EISCAT) observations of large ion outflows associated with the simultaneous occurrence of enhanced ion acoustic echoes. Although unavailable from the standard analysis procedure, the plasma description inside turbulent regions remains crucial to the understanding of the ion acceleration process related to enhanced ion acoustic echoes. However, their very clear spectral signatures make possible a more detailed approach to extract some information on the plasma behavior. This is what is attempted in Sect. 3. In Sect. 4, estimates of the electron temperature and of the ion drift obtained from the enhanced ion lines are used to study the possible implications on the plasma transport inside the turbulent regions.

\section{Observations}

EISCAT is an incoherent scatter radar facility in northern Scandinavia with VHF (224 MHz) and UHF (933 MHz) transmitters/receivers in Troms, Norway, and two additional UHF receivers in Kiruna, Sweden, and Sodankylä, Finland. This study uses data derived from the VHF radar which was pointed vertically. The VHF transmitter program, CP-7-D, used 900- $\mu$ s long pulses for measurement of the ion line autocorrelation functions in the altitude region 280 to $1600 \mathrm{~km}$, with a range resolution of $65.1 \mathrm{~km}$. Figure 1 presents $10 \mathrm{~s}$ integrated EISCAT data on May 2, 1991. It displays the height profiles from 280 to $1500 \mathrm{~km}$ of the electron density (panel 1), the electron and ion temperatures (panel 2 and 3) and the vertical ion velocity (panel 4) as observed by the VHF radar. For these data we have removed the plasma parameters when enhanced ion acoustic lines were present. Thus the turbulent regions appear as large data gaps (white areas). At high altitude, say above $1300 \mathrm{~km}$, white pixels appear because of the low signal to noise ratio. We see an occasionally strong $F$ region with maximum densities of about $10^{12} \mathrm{~m}^{-3}$ at 400-500 km (panel 1). Two enhanced electron temperature features (panel 2) associated with two ion outflows events (panel 4) can be seen around 133S and 1400 UT. Those structures can be identified as two auroral arc systems passing through the radar beam (Forme et al., 1995). Since the VHF radar makes an angle of about $13^{\circ}$ with the Earth's magnetic field line, a field-aligned structure (e.g., auroral arcs) passing the radar beam will trace a slope on our data due to the northward component of the velocity of the auroral structures.

The two arc systems are associated with high electron temperatures of about 6000 to $8000 \mathrm{~K}$, while the ion temperature remains unchanged except on the right edge of the first arc system, suggesting that a strong perpendicular electric field might be present there. This first arc is associated with electron temperatures of about $6000 \mathrm{~K}$ below the arc structure and decreasing above the turbulent region. This negative field-aligned temperature gradient (i.e., according to the slope traced by the arc) indicates that the heat source is located within or close to the turbulent region. On the other hand, the other arc system presents a positive field-aligned temperature gradient up to the uppermost altitude. The ion drift velocity (panel 4) associated with the first arc reaches extreme values of more than $1000 \mathrm{~m} / \mathrm{s}$ at $1000 \mathrm{~km}$ and decreases higher up suggesting that the acceleration mechanism is localized below $1000 \mathrm{~km}$.

Four turbulent structures (i.e. showing enhanced ion acoustic shoulders) that show quite different characteristics are present in our data between 1343 and 1352 UT. They do not show any of the typical characteristics of an auroral arc. They occur at an altitude of about $500 \mathrm{~km}$ and have a reduced altitude extent (100 to $200 \mathrm{~km})$ compared to the previously discussed cases. The associated electron temperature enhancements are more localized around these structures, and they do not give any indication of ion outflows. 

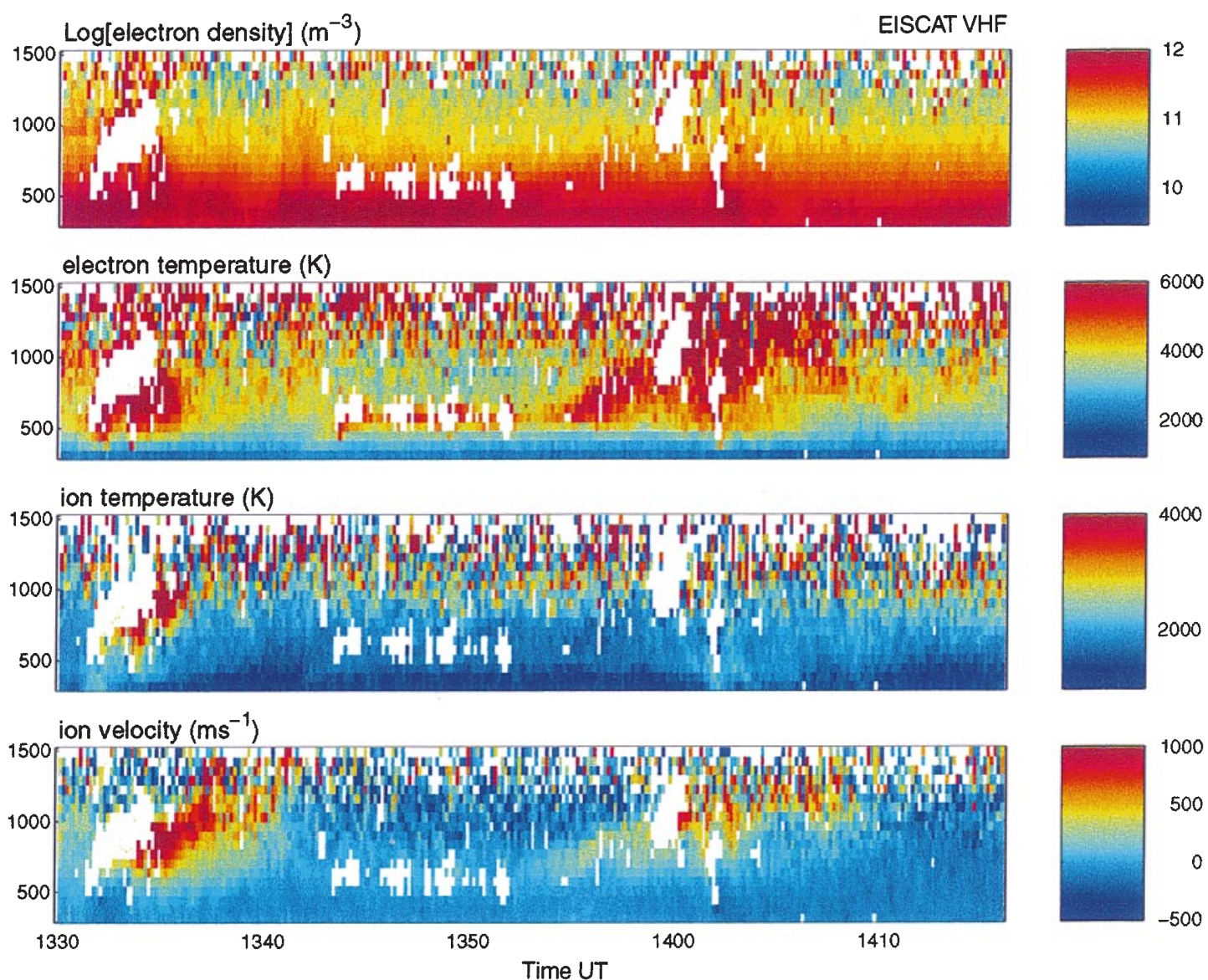

Fig. 1. Data derived from the VHF EISCAT radar during the time period 1330 to 1430 UT. Panel 1 shows an overview of the electron density, panel 2 the electron temperature, panel 3 the ion temperature

and panel 4 the vertical ion velocity (positive upward) between altitudes 280-1500 km (Forme et al., 1995)

\section{Estimates of plasma parameters inside the turbulent regions}

The auto-correlation functions (ACF) of the received signal are primary products of EISCAT measurements in the time domain. Beside the full analysis that fits the whole spectra, effective methods were developed to accurately estimate the plasma parameters from simple characteristics of the ACF shape such as maxima, minima, zero crossings. However, the severe signal distortions due to the enhanced acoustic echoes cause the failure of the classical analysis. It is beyond the scope of this work to discuss the somewhat complex steps of signal processing theories. We will rather remain at the level of basic and physical considerations concerning the frequency spectra, obtained by Fourier transform of the ACFs (which basically contain equivalent information in the frequency domain) and an intuitive interpretation in terms of waves.

\subsection{Usual and enhanced acoustic spectra}

The classical double-humped shape of an incoherent scatter spectrum is illustrated by the solid line in Fig. 2 as a function of the frequency shift relative to the transmitted frequency. Roughly, the spectral characteristics, equivalent to the $\mathrm{ACF}$ characteristics generally

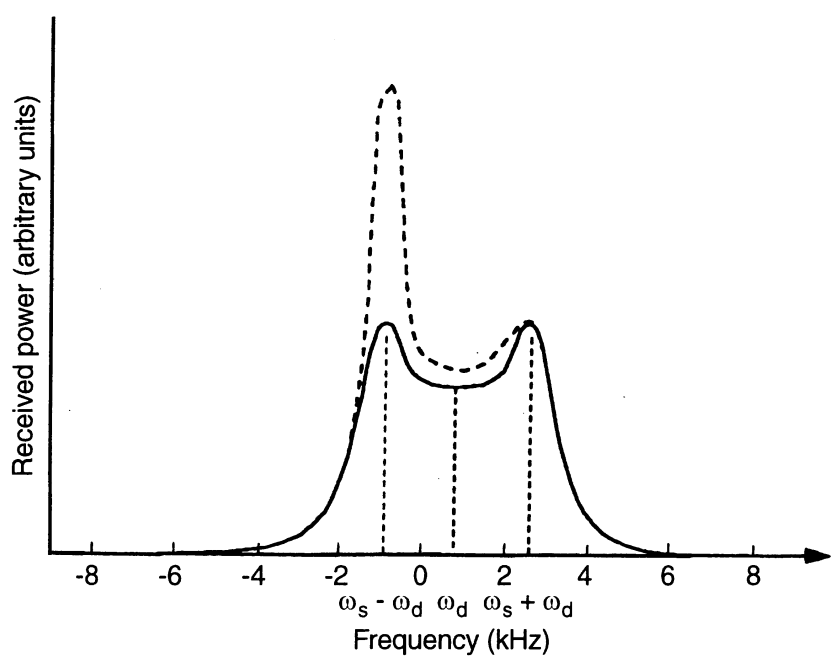

Fig. 2. Theoretical incoherent scatter spectra: classical doublehumped shape (solid line) and distorted shape due to ion acoustic fluctuations (dashed line) $\omega_{d}$ is the Doppler shift and $\omega_{s}$ the ion acoustic frequency 
used under appropriate assumptions to estimate the plasma parameters, are mainly:

1. The Doppler shift $\omega_{d}=\mathbf{k}_{s} \cdot \mathbf{V}_{d}$ of the whole spectrum, resulting from the ion bulk velocity $\mathbf{V}_{d}$ observed at the wave number $\mathbf{k}_{s}$ of the radar system;

2. The spectral width, related to the electron and ion temperatures $\left(T_{e}, T_{i}\right)$ and the mean ion mass $m_{i}$;

3 . The power ratio peak/valley, used to determine the ratio $T_{e} / T_{i}$;

4. And the total spectral power, proportional to the electron density $n_{e}$.

Moreover, if the mean ion mass is assumed, $T_{e}$ and $T_{i}$ can be easily determined by the spectral width and the peak to valley ratio. The dashed line in Fig. 2 shows the spectral distortion in the presence of acoustic echoes: the received power can be multiplied by 1 or 2 orders of magnitude, and the spectral shape becomes asymmetric with one acoustic line strongly enhanced and the other one also possibly modified. As a result, none of the characteristics mentioned can be identified, the standard analysis procedure fails and does not fit any plasma parameters.

\subsection{The method}

One may think that all the information on the plasma in an ISR spectrum is lost when ion acoustic fluctuations are enhanced. However, one feature at least seems to be preserved: the enhanced acoustic echoes are expected to occur at the frequency $\omega_{s}$ of the observed ion acoustic modes, propagating parallel and anti-parallel to the radar beam. The ion acoustic frequency is given by Forme et al. (1993):

$$
\begin{aligned}
\omega_{s}= & \frac{k_{s} \omega_{p, i}}{\sqrt{2} k_{D, e}\left(1+\frac{k_{s}^{2}}{k_{D, e}^{2}}\right)^{1 / 2}} \\
& \times\left[1+\left[1+12 \frac{T_{i}}{T_{e}}\left(1+\frac{k_{s}^{2}}{k_{D, e}^{2}}\right)\right]^{1 / 2}\right]^{1 / 2}
\end{aligned}
$$

where $k_{s}$ is the scattered wave vector imposed by the radar frequency, $\omega_{p, i}$ the ion plasma frequency, and $k_{D, e}$ the inverse of the electron Debye length. Also, the bulk frequency shift of both ion lines is not modified, and it is directly related to the Doppler shift, as illustrated in Fig. 2. Therefore only two measurements, the Doppler shift and the ion acoustic frequency, are available in the distorted spectra. The Doppler shift is a direct measurement of the ion velocity component along the line-ofsight, whereas the ion acoustic frequency depends on ion mass, electron and ion temperatures. Obviously, additional assumptions are thus required.

We first focus on the relevance of estimating two parameters and of fixing the others. EISCAT observations adjacent to turbulent regions mainly exhibit two remarkable features. The first one is a strong electron heating: electron temperature enhancements from about $3000 \mathrm{~K}$ up to $6000-8000 \mathrm{~K}$ are observed near acoustic echoes in F-region (Fig. 1), while the ion temperature remains almost unchanged (Rietveld et al., 1991; Wahlund et al., 1993; Forme et al., 1995). In addition, we have seen that a local maximum of the electron temperature profile suggests that the heating source is located inside the turbulent region. From simulations of the effects of low-frequency turbulence in the topside ionosphere, Forme et al. (1993) confirmed that the electron heating rate due to ion acoustic turbulence was predicted to exceed the ion heating rate by about one order of magnitude. The second remarkable feature observed near acoustic echoes is the occasional presence of intense field-aligned ion outflows: field-aligned velocities of more than $1000 \mathrm{~m} / \mathrm{s}$ were detected in the topside ionosphere (Wahlund et al., 1992b). Apart from these unusual enhancements in electron temperature and ion outflows, the other plasma parameters remain almost unchanged near turbulent regions. This has already been mentioned for the ion temperature, and is also the case for the electron density (Forme et al., 1995). We will assume that the ion composition remains unchanged. The only other noticeable variation does not occur near ion acoustic echoes, mostly observed in $F$-region, but at lower altitudes, where electron density enhancements in the $\mathrm{E}$ region suggest the correlated presence of precipitation.

Obviously, in the presence of ion acoustic echoes, two parameters may exhibit large variations departing from their usual level: the electron temperature and the ion field-aligned velocity. They are the best candidates to an estimate from the observed acoustic modes. We proceed as follows: (1) we determine the component of the ion drift velocity along the line-of-sight from the frequency shift of the maxima of the two acoustic lines relative to the radar frequency. This is certainly not the best method to estimate the Doppler shift, but, unlike more sophisticated and accurate methods, it does not involves any other characteristics of the spectrum shape, distorted by the presence of intense acoustic echoes. The resultant error in the Doppler shift depends on the frequency resolution of the observed spectrum. For our data set it is of the order of $300 \mathrm{~m} / \mathrm{s}$, much larger than the usual errors derived by accurate methods, when the standard analysis can be used. (2) The electron temperature is calculated from Eq. (1), and with the assumption that $k_{s}^{2} \lambda_{D, e}^{2} \ll 1$ (which is usually the case for the EISCAT VHF radar):

$T_{e}=\frac{\omega_{s}^{4} m_{i}^{2}}{2 k_{s}^{2} k_{b}\left(\omega_{s}^{2} m_{i}+6 k_{s}^{2} k_{b} T_{i}\right)}$

where $k_{b}$ is the Boltzmann constant and the ion temperature and the ion composition are assumed to keep their initial value just before the occurrence of the turbulence. For brief turbulent periods, say less than $1 \mathrm{~min}$, the ion temperature probably meets this assumption since the heating rate is much lower for ions than for electrons. This is more questionable for longer time periods, where the ion heating might vary, thus leading to errors in the determination of the electron temperature. These heating variations during turbulent events 
are taken into account by assuming an error as large as $100 \%$ on the value of $T_{i}$ estimated before the event. This will obviously lead to large errors on $T_{e}$, and such a method should rather be considered as a means to make a rough estimate of the plasma parameters at places where the analysis fails. Also the ion composition used was calculated from a 1-min integrated dump before the turbulence occurs. In the following we will take $10 \%$ error for the ion mass.

\subsection{Results}

We use the dataset presented in Sect. 2. The method presented will be applied to the first turbulent region observed between 1331:30 UT and 1335 UT. However, since the method presented can lead to large errors, we first check it on a non-turbulent case in order to test its validity. Then, we will use our method for a turbulent case and finally we will present large ion outflow events.

3.3.1 Validity check, 1330:10 UT. Figure 3 shows the electron temperature, the ion drift velocity and the observed spectra from 410 to $815 \mathrm{~km}$ altitude. Left panel shows the electron temperatures calculated from the ion acoustic frequency (solid line) and from the classical analysis (dashed line). The two curves fit rather well within the errors although the errors are large, about $800 \mathrm{~K}$. The same comments can be made for all dumps preceding the first appearance of the ion acoustic turbulence at 1331:30 UT. The ion drift, plotted in middle panel, also shows a good qualitative agreement between our estimate and the analyzed ion drift. The observed spectra (right panel) have the classical doublehumped shape, the total spectral power decreasing with
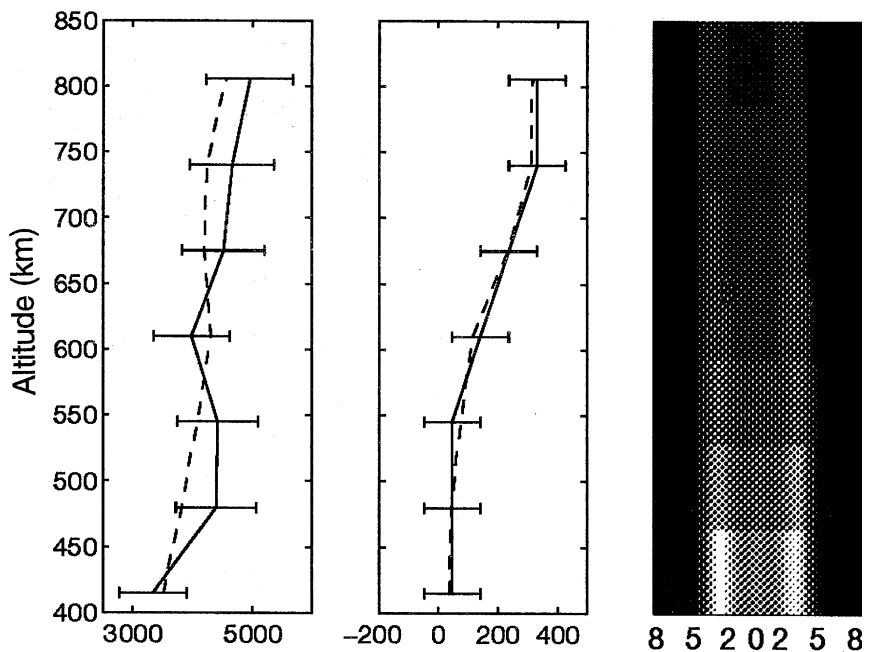

Fig. 3. Calculated electron temperatures from the ion acoustic frequency (solid line) and from the classical analysis (dashed line) at 1330:10 UT (left). Calculated ion vertical drift from the ion acoustic frequency (solid line) and classical analysis (dashed line) (middle). Color-coded spectra normalized to the maximum value (right) altitude as the density decreases. The Doppler shift used to calculate the ion drift velocity is almost imperceptible.

As expected, the determination of the parameter is less precise and involves larger errors by our method than by the classical analysis. Nevertheless, the trends of the profiles are globally correct and this encourages its application in disturbed cases when the analysis failed.

3.3.2 Turbulent case, 1331:30 UT. Figure 4 is an illustration of a turbulent case at the very beginning of the turbulence (see Fig. 1). Right panel shows that the spectra are enhanced from gate 2 (about $480 \mathrm{~km}$ ) to gate $4(610 \mathrm{~km})$, so that we do not plot the analyzed electron temperature and ion drift at those altitudes since they are not available from the standard analysis. The spectra show a clear asymmetry, the right acoustic shoulder being enhanced. Our estimate shows that while the electron temperature was about $4000 \mathrm{~K}$ before that dump, it increased up to $6000 \mathrm{~K}$ within 10 -s at the first turbulent gate (last panel). This reveals a very efficient heating rate for the electrons. Also the ion drift has been enhanced up to $400 \mathrm{~m} / \mathrm{s}$ just above the maximum of the electron temperature. The corresponding Doppler shift, of about one pixel, is seen in right panel.

3.3.3 Large ion outflows, 1335:00 and 1335:10 UT. In this section we will show two examples of large ion outflows, one within a turbulent region at 1335:00 UT, the second being when the turbulence stops at 1335:10 UT. The second example provides a good test to check once more the validity of our method since at that time plasma parameters derived by the standard analysis are available.

On Fig. 5 right panel, one can see that spectra from gate 3 to the highest gate are enhanced. The electron temperature (left panel) reaches a maximum of about $10000-11000 \mathrm{~K}$ at $675 \mathrm{~km}$ altitude, a very high value
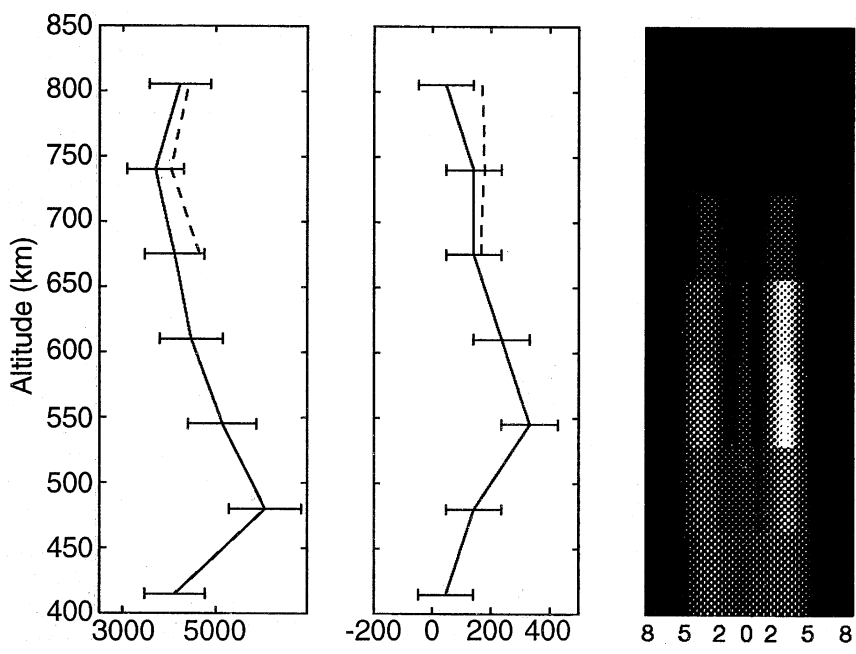

Fig. 4. Same as Fig. 3 at 1331:30 UT 

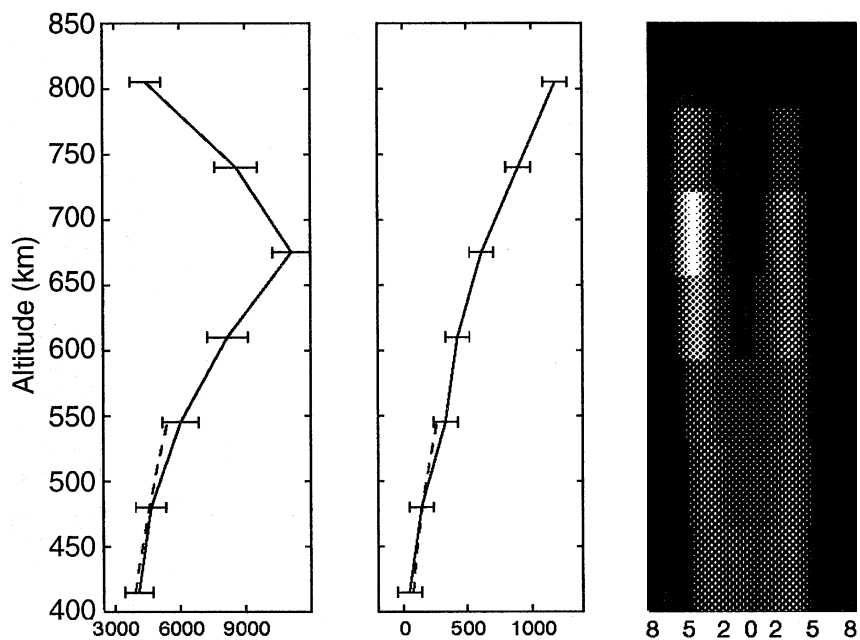

Fig. 5. Same as Fig. 3 at 1335:00 UT

indeed. The calculated ion drift, panel 2, increases up to $1200 \mathrm{~m} / \mathrm{s}$ at $800 \mathrm{~km}$. This behavior can be seen in right panel where a large Doppler shift increasing with altitude is seen on the observed spectra.

10-s later, the observed spectra show a classical behavior (decreasing amplitude with increasing altitude) but still present a large Doppler shift (Fig. 6 right panel). Although plasma parameters derived by the standard analysis are now available, we have used the ion temperature and density profile used in the previous example to calculate $T_{e}$ and the momentum terms. We want to show that our assumption that $T_{i}$ and $n_{e}$ are slowly varying is valid in our case. The ion drift has now reached $1300 \mathrm{~m} / \mathrm{s}$ at $800 \mathrm{~km}$ and the calculated drift fits the analyzed ion drift rather well (Fig. 6 middle panel). Ion fluxes reach up to $2.310^{14} \mathrm{~m}^{-2} \mathrm{~s}^{-1}$ at the highest altitude. The same conclusion can be drawn concerning the electron temperature, but it has decreased to less than $8000 \mathrm{~K}$ (left panel).
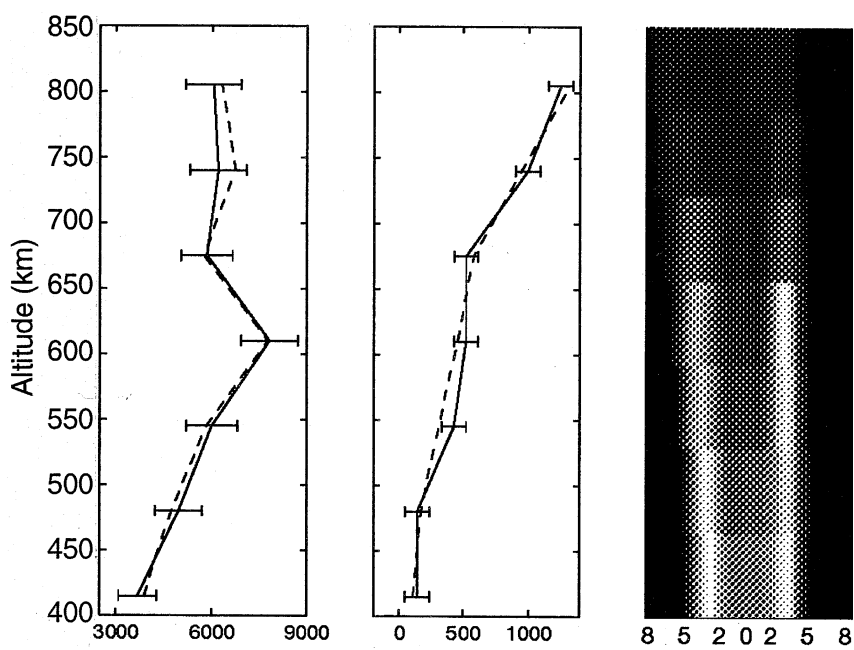

Fig. 6. Same as Fig. 3 at 1335:10 UT

\section{Discussion}

The method presented in Sect. 3 allows us to estimate very high electron temperatures (up to $11000 \mathrm{~K}$ ) when enhanced ion-acoustic fluctuations are observed. Temperatures of about 6000 to $8000 \mathrm{~K}$ corresponding to enhanced ion acoustic fluctuations have already been reported by Wahlund et al. (1993) and Forme et al. (1995). However, those values were observed adjacent to the turbulent regions. Hence, temperatures as high as 9000 to $11000 \mathrm{~K}$ are not so surprising when and where the turbulence acts as an additional heat source. Indeed, such very high electron temperatures were inferred from a theoretical approach by Forme et al. (1993). They showed that the topside ionosphere can be heated up to 1 or $2 \mathrm{eV}$ if strong field-aligned currents can trigger the ion-acoustic instability. The same authors predicted a sharp electron temperature gradient of about $0.02 \mathrm{~K} \mathrm{~m}^{-1}$. The data presented in the previous section also show a strong temperature gradient of 0.01 to $0.02 \mathrm{~K} \mathrm{~m}^{-1}$ (e.g., Fig. 4). EISCAT data show regions of enhanced ion acoustic fluctuations where no ion outflows are detected. Those regions are characterized by a relatively low heating rate due to the turbulence. On the other hand, the largest ion outflows occur where the electrons are strongly heated by the turbulence. This suggests that the electron temperature is of importance to understand the acceleration mechanism. Wahlund et al. (1992b) suggested that the ions are accelerated by a parallel electric field in the ionosphere. In their interpretation the enhanced field-aligned electric field is caused by anomalous resistivity due to low-frequency plasma turbulence. They contrasted this type of acceleration mechanism with the thermal ion outflows where frictionally heated ions cause strong pressure gradients, which in turn push them outward. Since we have an estimate of the electron temperature and the ion drift velocity inside the turbulent regions it is now possible to calculate the ion momentum equation to understand the implication of sharp electron temperature increases on the ion dynamics. The steady state vertical momentum equation for ions, neglecting field-aligned currents and the vertical neutral velocity can be written as (Schunk and Walker, 1970):

$$
\begin{aligned}
v_{z} \frac{\partial v_{z}}{\partial z} & +\sin ^{2} I\left(\frac{1}{m_{i} n_{i}} \frac{\partial}{\partial z}\left(n_{i} k_{b} T_{i}\right)+\frac{e}{m_{i}} E-g\right) \\
& +v_{z} \nu_{i n}=0
\end{aligned}
$$

where $g$ is the gravity field strength, $n$ the density, $v_{z}$ the vertical velocity, $\mathrm{m}$ the particule mass, $z$ the altitude, $I$ the magnetic dip angle and $E$ the charge separation electrostatic field given by:

$E=\frac{1}{e n_{e}} \frac{\partial}{\partial z}\left(n_{e} k_{b} T_{e}\right)$.

To calculate Eq. (3) inside the turbulent regions we will assume that the ion temperature keep its initial value just before the occurrence of the turbulence. Also, the electron density is unknown and it has not been estimated by the method presented in Sect. 3. Therefore, 
as for the ion temperature, the electron density is taken from the last non-turbulent profile, assuming that it changes over a much larger time scale than the electron temperature. This approximation can be checked by the use of the continuity equation:

$\frac{\partial n_{e}}{\partial t}+\frac{\partial n_{e} v_{z}}{z}=0$

for strong ion outflows (e.g., Fig. 6). The time scale corresponding to a $10 \%$ variation of $n_{e}$ is of about $1 \mathrm{~min}$. Since the turbulence lasts at most for about 3 min we will assume a $30 \%$ error on $n_{e}$. The ions are assumed to be $0^{+}$, ion neutral collision frequencies are taken from Schunk and Nagy (1980), and the neutral temperature and densities are taken from the MSIS86 model.

Figure 7 shows the result of calculation of Eq. (3) at times 1330:10 UT, 1331:30 UT and 1335:10 UT corresponding to the ion drift velocity and electron temperature plotted on Fig. 3, 4 and 6, respectively. Two curves are displayed, one representing the advection and the collisional terms (solid line), the other one representing the pressure gradients and the gravitational terms (dashed line). The source term being the pressure gradients, a good fit between the two curves indicates that Eq. (3) is balanced and therefore that pressure gradients can explain the observed velocity profile.

At 1330:10 UT it is seen that pressure gradient sources are enough to explain the velocity profile seen in Fig. 3 above $450 \mathrm{~km}$. At 1331:30 UT the two curves fit rather well above $500 \mathrm{~km}$ indicating that the sudden increase of the electron temperature seen on Fig. 4 can explain the ion drift above $500 \mathrm{~km}$. Below that altitude, it seems that more momentum input is needed. Indeed the pressure gradient sources become negative thus downward ion motion should be expected while small
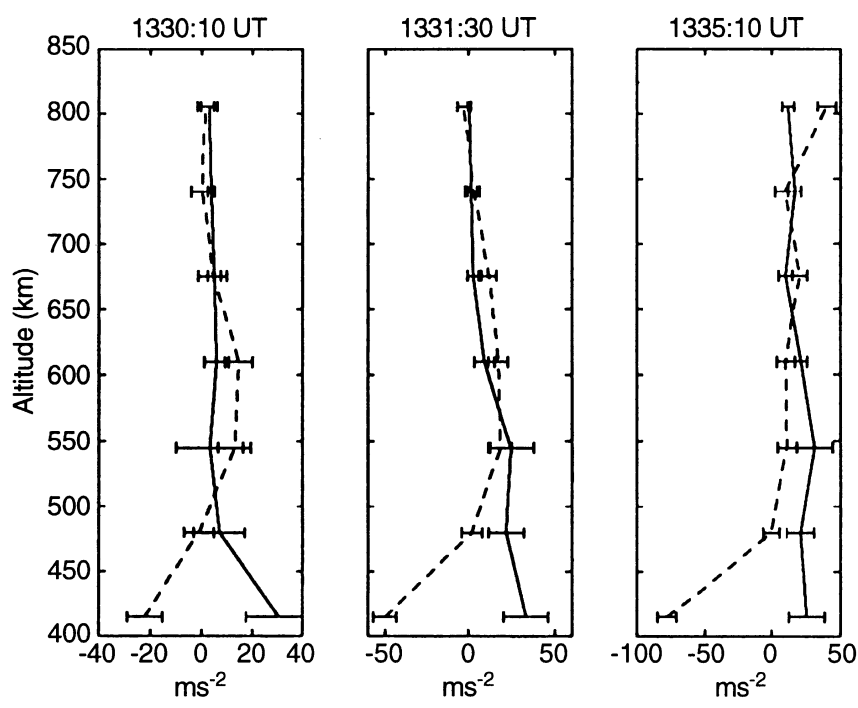

Fig. 7. Calculated momentum terms at three different times 1330:00 UT, 1331:30 UT and 1335:10 UT. The solid lines represent the advection combined with the ion-neutral collisional term. The dashed line is the combination of the pressure gradients and the gravitational terms ion upflows are observed below $500 \mathrm{~km}$. This discrepancy can be explained in terms of neutral atmospheric motions which drag the plasma to small field aligned velocities of about a few tens of $\mathrm{m} \mathrm{s}^{-1}$. For the large ion outflow seen at 1335:10 UT once again the pressure gradient term is comparable to the collisional term above $500 \mathrm{~km}$. Obviously, we do not need any additional mechanism to accelerate the ions upward. Since, in our calculation, the ion temperature profile was kept constant between 1331:30 UT and 1335:10 UT, the ion acceleration is mainly due to the electron temperature increase which causes a large electron pressure gradient. This mechanism differs from the classical thermal ion outflows where ions are frictionally heated when they move through the neutral gas under the action of a strong perpendicular electric field. Indeed, the electrons are firstly heated which enhances the ambipolar electric field that finally pushes the ions outward. It should be mentioned that neglecting the neutral motion increases the collisional term. On the other hand, assuming that the ion temperature remains unchanged, while we know that ions are heated, minimizes the estimation of the ion pressure gradient term. For these reasons, it is believed that the collisional term is over-estimated while the pressure gradient is under-estimated.

\section{Conclusions}

We have reported EISCAT VHF observations of enhanced ion acoustic fluctuations. It was shown that when turbulent regions are characterized by strong electron heating then large ion outflows exist with ion fluxes of about $10^{14} \mathrm{~m}^{-2} \mathrm{~s}^{-1}$. On the other hand turbulent regions with lower electron heating do not show any ion outflows. The electron temperature profiles show local maxima indicating that the heat source is located within the turbulent regions. Enhanced ion acoustic fluctuations cause the failure of the standard analysis which deprives us of the plasma parameters inside turbulent regions. However, even rough estimates of the plasma parameters are of crucial importance to understand the thermal and dynamic behavior of the ionospheric plasma when enhanced ion acoustic fluctuations are detected. We have proposed a method to determine the ion drift velocity and the electron temperature assuming that all other parameters, namely the electron density, the ion temperature and the ion composition remain constant. This method is based on the fact that the ion acoustic frequency and the Doppler shift are not modified by the enhanced ion acoustic fluctuations. We have checked the validity of our method by comparing its results with parameters derived from the standard analysis. Although it leads to large errors, this method gives a good quantitative agreement with the standard analysis. Applied within the turbulent regions, it shows that electrons are strongly heated up to $11000 \mathrm{~K}$ and that sharp electron temperature gradients of $0.02 \mathrm{~K} / \mathrm{m}$ exist at altitudes of about 500 to $800 \mathrm{~km}$. Simultaneously, ions reach velocities up to $1300 \mathrm{~m} / \mathrm{s}$ at $800 \mathrm{~km}$. Implications of 
such a strong electron heating have been studied by using the vertical ion momentum equation. It is suggested that the strong electron temperature gradients estimated from the observations can increase the ambipolar electric field, resulting in large ion outflows.

Acknowledgements. We are grateful to the Director and staff of EISCAT for their help. EISCAT is supported by the Research Council of France (CNRS), West Germany (MPG), Norway (NAVF), Sweden (NFR), Finland (SA) and the UK (PPARC). Topical Editor D. Alcaydé thanks M. Rietveld and A. Aylward for their help in evaluating this paper.

\section{References}

Forme, F. R. E., A new interpretation on the origin of enhanced ion acoustic fluctuations in the upper ionosphere, Geophys. Res. Lett., 20, 2347, 1993.

Forme, F. R. E., J. E. Wahlund, H. J. Opgenoorth, M. A. L. Persson, and E. V. Mishin, Effects of current driven instabilities on the ion and electron temperatures in the upper ionosphere, $J$. Atmos. Terr. Phys., 55, 647, 1993.

Forme, F. R. E, D. Fontaine, and J. E. Wahlund, Two different types of enhanced ion acoustic fluctuations observed in the upper ionosphere, J. Geophy. Res., 100, 14 625, 1995.

Chappell, C. R., T. E. Moore, and J. H. Waite, Jr., The ionosphere as a fully adequate source of plasma for the Earth's magnetosphere, J. Geophys. Res., 92, 5896, 1987.

Collis, P. N., I. Häggström, K. Kaila, and M. T. Rietveld, EISCAT radar observations of enhanced incoherent scatter spectra; their relation to red aurora and field-aligned currents, Geophys. Res. Lett., 18, 1991.

Farmer, A. D., M. Lockwood, R. B. Horne, B. J. I. Bromage, and K. S. C. Freeman, Field-perpendicular and field-aligned flows abserved by EISCAT during a prolonged period of northward IMF, J. Atmos. Terr. Phys., 46, 473, 1994.
Foster, J. C., C. del Pozo, K. Groves, and J. -P. St.-Maurice, Radar observations of the onset of current driven instabilities in the topside ionosphere, Geophys. Res. Lett., 15, 160, 1988.

Johnson, R. G., Energetic ion composition in the Earth's magnetosphere, Rev. Geophys., 17, 696, 1979.

Jones, G. O. L., P. J. S. Williams, K. J. Winser, M. Lockwood, and K. Suvanto, Large plasma velocities along the magnetic field line in the auroral zone, Nature, 336, 231, 1988.

Lockwood, M., J. H. Waite Jr., T. E. Moore, J. F. E. Johnson, and C. R. Chappell, A new source of suprathermal $\mathrm{O}^{+}$ions near the dayside polar cap boundary, J. Geophys. Res., 90, 4099, 1985.

Rietveld, M. T., P. N. Collis, and J. P. St.-Maurice, Naturally enhanced ion acoustic waves in the auroral ionosphere observed with EISCAT $933 \mathrm{MHz}$ radar, J. Geophys. Res., 96, 19 291, 1991.

Schunk, R. W., and A. F. Nagy, Electron temperatures in the F region of the ionosphere: theory and observations, Rev. Geophys. Space. Phys., 16, 355, 1978.

Schunk, R. W., and J. C. G. Walker, Transport properties of the ionosphere electron gas, Planet. Space. Sci., 18, 1535, 1970.

Wahlund, J. E., F. R. E. Forme, H. J. Opgenoorth, M. A. L. Persson, E. V. Mishin, and A. S. Volokitin, Scattering of electromagnetic waves from a plasma: enhanced ion acoustic fluctuations due to ion-ion two-stream instabilities, Geophys. Res. Lett., 19, 1919, 1992a.

Wahlund, J. E., H. J. Opgenoorth, I. Häggström, K. J. Winser, G. O. L. Jones, EISCAT observation of topside ionospheric ion outflows during auroral activity: revisited, J. Geophys. Res., 97, 3019, 1992b.

Wahlund, J. E., H. J. Opgenoorth, F. R. E. Forme, M. A. L. Persson, I. Häggström, and J. Lilensten, Electron energization in the topside auroral ionosphere: the importance of ion-acoutic turbulence, J. Atmos. Terr. Phys., 55, 623, 1993.

Winser, K. J., G. O. L. Jones, P. J. S. Williams, and M. Lockwood, Observations of large field-aligned flows of thermal plasma in $\mathrm{t}$ he auroral ionosphere, Adv. Space Res., 5, 57, 1989. 